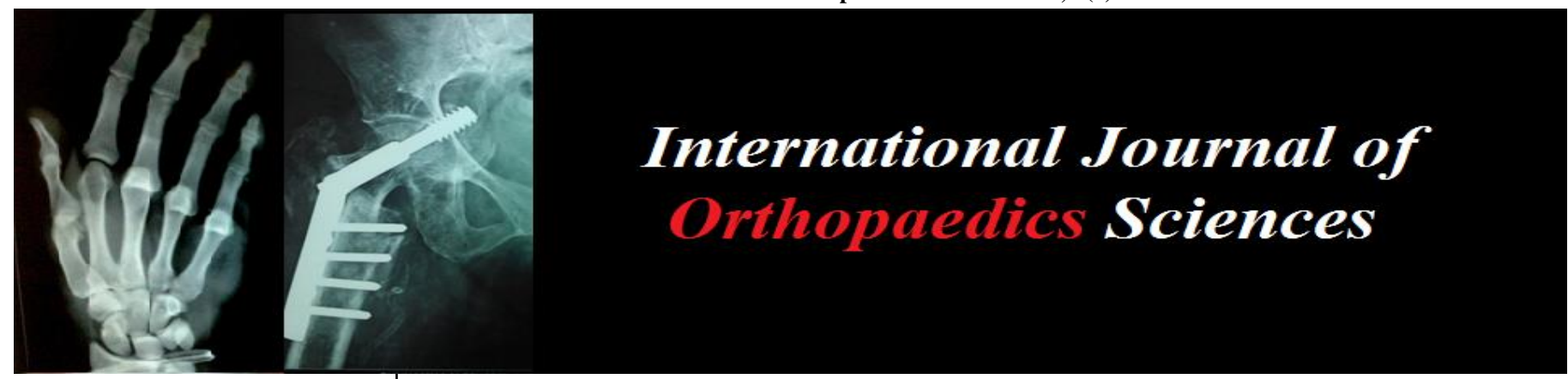

E-ISSN: 2395-1958

P-ISSN: 2706-6630

IJOS 2021; 7(4): 188-190

(C) 2021 IJOS

www.orthopaper.com

Received: 21-06-2021

Accepted: 23-07-2021

Dr. Rohan Parwani

Assistant Professor, Department of Orthopedics, PDU Medical

College, Rajkot, Gujarat, India
Corresponding Author: Dr. Rohan Parwani

Assistant Professor, Department of Orthopedics, PDU Medical College, Rajkot, Gujarat, India

\section{The spectrum of septic hip arthritis in neonates, a study of 12 children}

\section{Dr. Rohan Parwani}

DOI: https://doi.org/10.22271/ortho.2021.v7.i4c.2883

\section{Abstract}

Septic arthritis is a common reason for persistent sepsis in neonates. It is often a result of systemic sepsis. While the treatment is clear in older children, with majority of them requiring surgical intervention, the high risk of anesthesia in small children makes surgical intervention tricky. We studied various parameters in 12 children that presented to us for management of septic arthritis. We found that a timely surgical intervention, even if there is a mild effusion leads to a shorter IV antibiotic requirement and early return of motion.

Keywords: hip arthritis, septic arthritis, neonates, NICU

\section{Introduction}

Pediatric neonatal septic arthritis is a dreaded complication of neonatal sepsis ${ }^{2}$. Neonatal sepsis happens in preterm and term children due to various reasons that include puerperal sepsis, respiratory distress syndrome, hypoglycemia, etc. ${ }^{[1]}$. The sepsis is initially often blood borne. But as the immunity and iv antibiotics respond, the septicemia is converted into deeper infections that are often hard to get to and have restricted blood supply.

The increased relative vascularity of synovium makes it susceptible to infection. Once the infection sets in, the synovium, there is inflammation and neutrophilic exudate. As the joint is a closed cavity there is poor antibiotic penetration into the collection. There remains persistence of bacteria in the collection and soon this collection becomes resistant to systemically administered IV antibiotic. Septic arthritis can be treated with IV antibiotics only or with surgical debridement ${ }^{[2]}$. The decision to operate or not is usually based on ultrasound or other radiological studies. The decision to operate is often complicated by a high risk of anesthesia in such children and getting parental consent for surgery is often difficult ${ }^{[2]}$. Missed or late presenting septic arthritis also has a devastating effect on the development of joints and this affects future mobility ${ }^{[1]}$. Inadequately treated septic arthritis can easily turn into osteomyelitis or have a long-standing effect on the joint and predisposes the child to multiple future interventions ${ }^{[1]}$. The presence of collection usually requires surgical debridement. However, in the current scenario with easy availability of improved perinatal care, fulminant septic arthritis is rare ${ }^{[3]}$. In our experience, most septic arthritis seen by orthopedic surgeons today happens in NICU where the child is already under neonatal care and receiving IV antibiotics. Deciding on such a scenario becomes tricky because the inflammatory markers are rarely high to indicate bad sepsis and often there is a minimal collection ${ }^{3}$. Our study attempted to define the role of surgical treatment, in the management of neonatal septic arthritis in a NICU scenario.

\section{Aims and objectives}

To identify indications of operating neonatal septic arthritis.

To identify the causative organisms usually seen in neonatal septic arthritis.

To study the change in management after surgical intervention in neonatal septic arthritis.

To study the change in the duration of IV antibiotics after surgical intervention.

To study the outcome of surgical intervention.

To study the microorganism responsible for septic arthritis. 


\section{Materials and methods}

After due ethical approval, subjects were included meeting the following criteria.

\section{Inclusion criteria}

The child admitted to NICU, before 1 month of age

Age at presentation less than 1 month of age

\section{Exclusion criteria}

Other joint septic arthritis.

Septic arthritis is seen after discharge at home.

All septic arthritis that presented to us from August 2019 to August 2020 was included in the study. All the septic arthritis was seen and managed by the lead author. We collected the following data from each patient.

Age (days)

Sex

Birth weight $(\mathrm{kg})$

Reason for NICU Admission

Day of Onset of Hip pain

IV Antibiotics administered previously

Flexion Attitude

Fever

Swelling

Other joint involvement

Total Count

CRP

ESR

USG

Blood Culture

Surgical Debridement

IV Antibiotics

Duration of Post-op Antibiotics

Synovial Fluid Culture

Time to recovery of spontaneous movement

6-month followup

After due consent, septic arthritis surgery was performed in septic operation theatre. Under short general anesthesia, $2 \mathrm{~cm}$ incision was made centered on hip joint, $1-1 / 2 \mathrm{~cm}$ anteromedial to ASIS. Plane was developed between sartorius and tensor fascia lata. Reflected head of rectus femoris is elevated and capsule identified. First, with a thick gauge needle, intraarticular fluid is aspirated. This is followed by 1by $1 \mathrm{~cm}$ capsular window. Thorough wash with saline and hydrogen peroxide is given. Capsule is left open over a drain and skin closure ois done. Material from inside the joint is sent for histopathological examination and culture to identify the organism.

\section{Results}

Our study included 12 children, who presented to us over one year. The lead author is attached, visiting surgeon to multiple NICU. All the children who are included over one year are included in this study.

The mean age of presentation of septic arthritis to the author was 34 days. Two children presented at a very young age (5 and 9 days), where they had septic arthritis as a component of primary neonatal-perinatal sepsis. Ten children were admitted to NICU for alternate diagnosis and as a part of their neonatal stay, developed sepsis which localized to septic arthritis of the hip. The mean birth weight was $2.4 \mathrm{~kg}$ in all kids. There were various reasons for admission in NICU with 4 out of 12 kids having respiratory distress syndrome. One kid was admitted for low birth weight and 5 kids were admitted for primary sepsis. Of all the patients, only two kids had primary septic arthritis on admission, the other ten kids developed septic arthritis after a short duration of stay in NICU, during which IV antibiotics were already administered. Blood culture for the choice of IV antibiotics was positive in 10 kids, staphylococcus aureus is the most common organism in 7 kids, and e coli has been detected in two kids, with no result in two kids.

The common presenting complaint, when an orthopedic opinion was sought was persistent flexion attitude of the hip. Fever was present in only 2 out of 12 kids. No external swelling was detected and multiple joint involvements were found in only 4 out of 12 kids. Total count, CRP was elevated in all kids, with mean values being 18,818, total count and mean of $65 \mathrm{CRP}$ and $117 \mathrm{ESR}$. Notable exceptions were there, where septic arthritis presented with a relatively low CRP of 38 and 35 in two kids and an almost normal total count of 12600 and 13300 in two kids. Radiological examination showed mild effusion in nine kids and moderate effusion in the other three kids.

Surgical intervention was performed in 7 out of 12 kids of which four had moderate effusion and three had mild effusion. Kids with mild effusion were offered surgical intervention because of poor response to empirical IV antibiotics. In kids, who underwent surgical debridement a minimum of $4 \mathrm{ml}$ thick fluid was found. In two kids who had primary septic arthritis at presentation, $8 \mathrm{ml}$ frank pus was found. The choice of IV antibiotics was guided by the synovial fluid culture and sensitivity. The most frequently isolated organism was Staphylococcus aureus, with all of them being MRSA and responding to vancomycin meropenem combination. One child had candida isolated in his fluid and was given Amphotericin B for 30 days. Two children had e coli isolated in culture and had meropenem and linezolid IV antibiotics. The duration of IV antibiotics was 14 days in almost all operated kids, while kids managed nonoperatively needed a longer duration of IV antibiotics. After a long follow-up of 6 months, only one kid who presented to us with primary septic arthritis had absent epiphysis but a located hip. The rest of the kids had normal hip development and movement at 6 months follow-up. The mean time to recovery of spontaneous moments was 26 days, with early recovery of movement seen in kids with surgical intervention and delayed recovery of movement, seen in kids who underwent IV antibiotic treatment only.

\section{Discussion and conclusion}

Our study was performed to give a brief outline of the clinical scenario in which neonatal septic arthritis is seen in today's practice, where improved NICU care is easily available. Improved availability of NICU care has meant that all highrisk babies are kept under observation. At the earliest indication of raise of inflammatory markers, IV antibiotics are started and this prevents development of gross sepsis. Also many LBW children, who do not accept oral intake are maintained on IV parenteral nutrition for initial days. Presence of an IV line, predisposes them to sepsis. Our study has concluded that primary septic arthritis is rare but best treated with surgical intervention. But most of septic arthritis that we see in this age group happens in children who already have received a substantial dose of empirical IV antibiotics and as such the volume of effusion is low. Securing and maintaining intravenous lines in such low-birth-weight neonates is a problem and we must try to reduce the duration of IV antibiotics which also have systemic complications. 
From this study, we concluded that offering an early surgical intervention with due parental consent and in children who are not responding to IV antibiotics will help shorten the duration of IV antibiotics and lead to an earlier return of movement. The organism isolated, can be different from the organism isolated from blood culture. The organism is often related to the type of bacteria usually found in the NICU environment. Even after full management of septic arthritis is complete, there is residual flexion attitude in child in hip which takes some time to improve.

\section{References}

1. Samora JB, Klingele K. Septic arthritis of the neonatal hip: acute management and late reconstruction. J Am Acad Orthop Surg 2013;21(10):632-41. doi: 10.5435/JAAOS-21-10-632. PMID: 24084437.

2. Rai A, Chakladar D, Bhowmik S et al. Neonatal septic arthritis: Indian perspective. Eur J Rheumatol. 2020;7(Suppl 1):S72-S77. doi:10.5152/eurjrheum.2019.19052

3. O'Meara PM, Bartal E. Septic arthritis: process, etiology, treatment outcome. A literature review. Orthopedics. 1988;11(4):623-8. PMID: 3290872. 